\title{
A Rare Case of Angioleiomyoma Arising in the Subglottic Area to Upper Trachea of a Patient with Underlying Asthma
}

Yeoun Eun Sung · Chin Kook Rhee ${ }^{1}$ Kyo Young Lee

Department of Hospital Pathology, ${ }^{1}$ Division of Pulmonary, Allergy and Critical Care Medicine, Department of Internal Medicine, Seoul St. Mary's Hospital, College of Medicine, The Catholic University of Korea, Seoul, Korea

Received: June 2, 2016

Revised: June 15, 2016

Accepted: June 21, 2016

\section{Corresponding Author}

Kyo Young Lee, PhD

Department of Hospital Pathology, College of

Medicine, The Catholic University of Korea, 222

Banpo-daero, Seocho-gu, Seoul 06591, Korea

Tel: +82-2-2258-1618

Fax: +82-2-2258-1627

E-mail: leekyoyo@catholic.ac.kr
Angioleiomyoma is a rare disease that is histologically characterized by smooth muscle cells arranged around vascular spaces. Although angioleiomyomas occur rarely in the head and neck region, they can cause various symptoms according the site involved. Here, we present a 44-yearold male patient with a 15-year history of asthma, who presented with recent onset of chest discomfort, globus sensation and throat pain. Medication was not effective in relieving his symptoms, and further evaluation revealed a polypoid ovoid mass, almost obstructing the airway at the border of the larynx and upper trachea on chest computed tomography. The mass was completely resected via a rigid bronchoscopy procedure. Histopathologic examination revealed that the excised mass was angioleiomyoma, which was immunohistochemically positive for smooth muscle actin and negative for desmin.

Key Words: Angioleiomyoma; Larynx; Trachea; Bronchoscopy
Angioleiomyoma or vascular leiomyoma is a benign dermal or subcutaneous tumor composed of well-differentiated smooth muscle cells arranged around many vascular channels. ${ }^{1}$ It can occur anywhere in the body but is most often seen in the extremities, particularly the lower leg. ${ }^{2}$ Angioleiomyoma of the head and neck region is uncommon ( 10\%), with lips, ears, and nose being the most frequently involved locations. ${ }^{2,3}$ Here, we present a case of angioleiomyoma at the border of the subglottic area and upper trachea, with a clinical and pathologic approach.

\section{CASE REPORT}

A 44-year-old man presented to the pulmonology department of our hospital for a one week history of chest discomfort, globus sensation, and throat pain. The patient had a history of asthma for 15 years with about 10 events a year but was not taking any medication. He was prescribed a leukotriene receptor antagonist, an inhaled corticosteroid plus long acting beta agonist and a long acting muscarinic antagonist to control bronchial asthma for 3 months. Although his pulmonary function test results showed improvement, the patient persistently complained of globus sensation and throat pain with slight aggravation. Therefore, he was referred to an otolaryngologist for evaluation of his throat and vocal cords.

Laryngoscopy revealed a subglottic mass, which was almost completely occluding the airway. Computed tomography revealed a $2.0 \times 1.5-\mathrm{cm}$-sized, round exophytic lesion at the border of the subglottic area and upper trachea (Fig. 1A, B). The lesion was well-enhancing and adjacent structures showed no evidence of involvement or destruction. Separately, a $3.0 \times 2.6 \mathrm{~cm}$ pituitary mass suggestive of a macroadenoma was also incidentally found.

Excision of the mass was performed by interventional pulmonologists and cardiothoracic surgeons via rigid bronchoscopy. The mass was located $1 \mathrm{~cm}$ below the vocal cord, attached to the anterior wall of the trachea by a stalk-like structure (Fig. 1C). It was mobile and resected by snare without any complications (Fig. 1D).

After surgery, the tissue was sent to the pathology department for diagnosis. On gross examination, the tumor was a solid, 

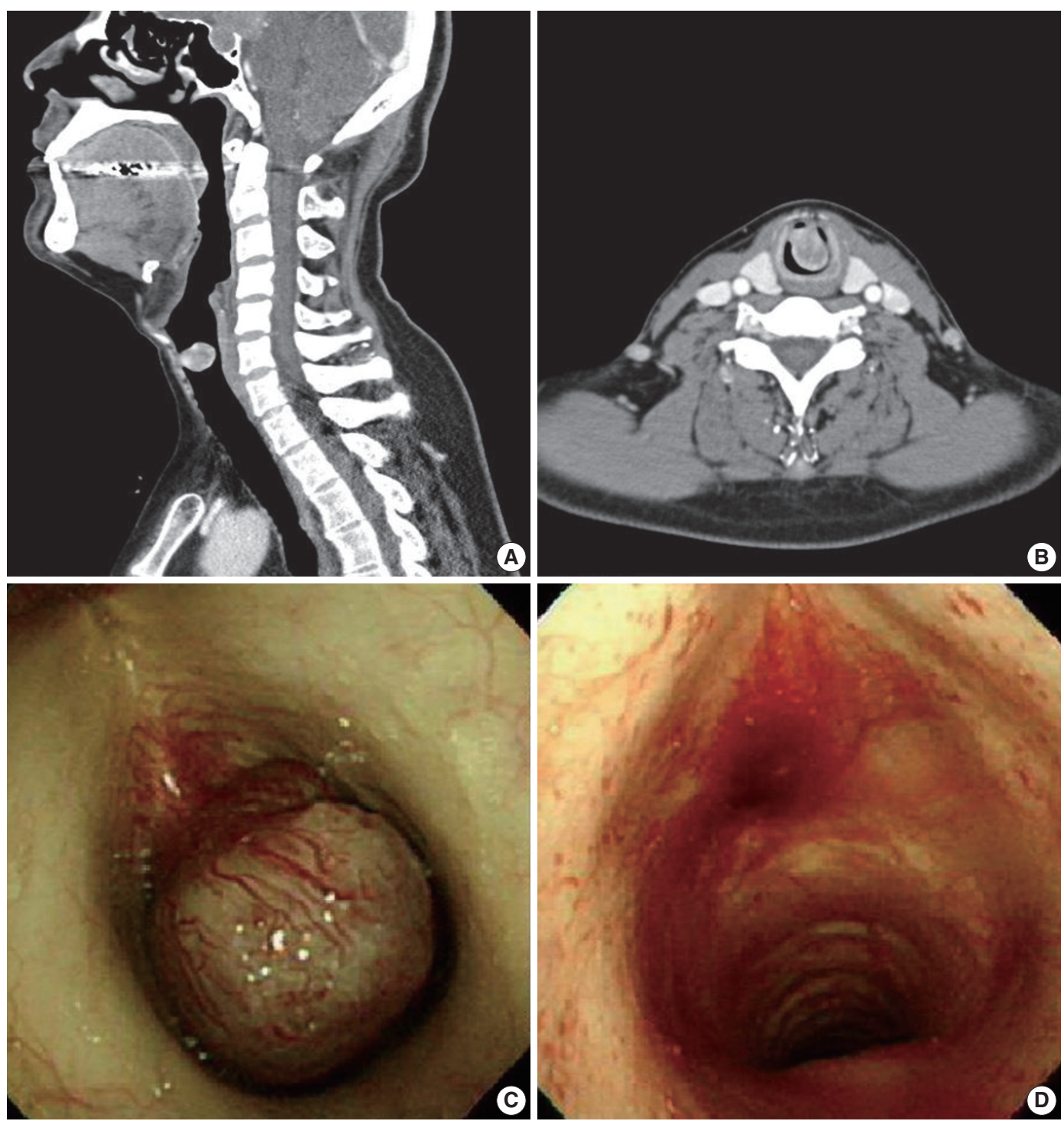

Fig. 1. Neck computed tomography (CT) and bronchoscopic findings. (A, B) Neck CT reveals a 2.0 ×1.5-cm-sized, round exophytic lesion at the border of the subglottic area and upper trachea. (C) Bronchoscopic finding during the operation, showing an ovoid and polypoid mass located $1 \mathrm{~cm}$ below the vocal cord, attached to the anterior wall of the trachea by a stalk-like structure. (D) Bronchoscopic view of the same lesion after the excision; the mass is resected by snare without complications.

ovoid, and polypoid mass with a firm consistency, measuring $1.6 \times 1.4 \times 1.0 \mathrm{~cm}$. It had a grayish and whirling cut surface. Prominent vessels were noticeable on the surface, which resembled feeding vessels as they were thick and more abundant near the resected stalk and spreading branches.

Histologically, the mass consisted of well differentiated smooth muscle cells with intervening vascular channels (Fig. 2A). These channels were mostly venous with variable venous lumens surrounded by muscular coats with dense to relatively loose intervascular smooth muscle cells (Fig. 2B). The muscular coats surrounding the vessels were of variable thickness and some portion of the intervascular stroma was edematous with very sparsely placed smooth muscle bundles. The cells were bland and no mitosis was found (Fig. 2B). A few foci of aggregated adipocytes and mild lymphocytic infiltration were also noted. The surface was covered with ciliated pseudostratified columnar bronchial epithelium (Fig. 2C). Thin walled vessels were abundantly placed in the lamina propria, some of which had an ill-defined muscular coat (Fig. 2C). Immunohistochemically, the tumor cells were positive for smooth muscle actin (Fig. 2D) and negative for desmin.

The patient did not develop any complications after surgery and showed no residual tumor or abnormal lesion in follow-up studies. 

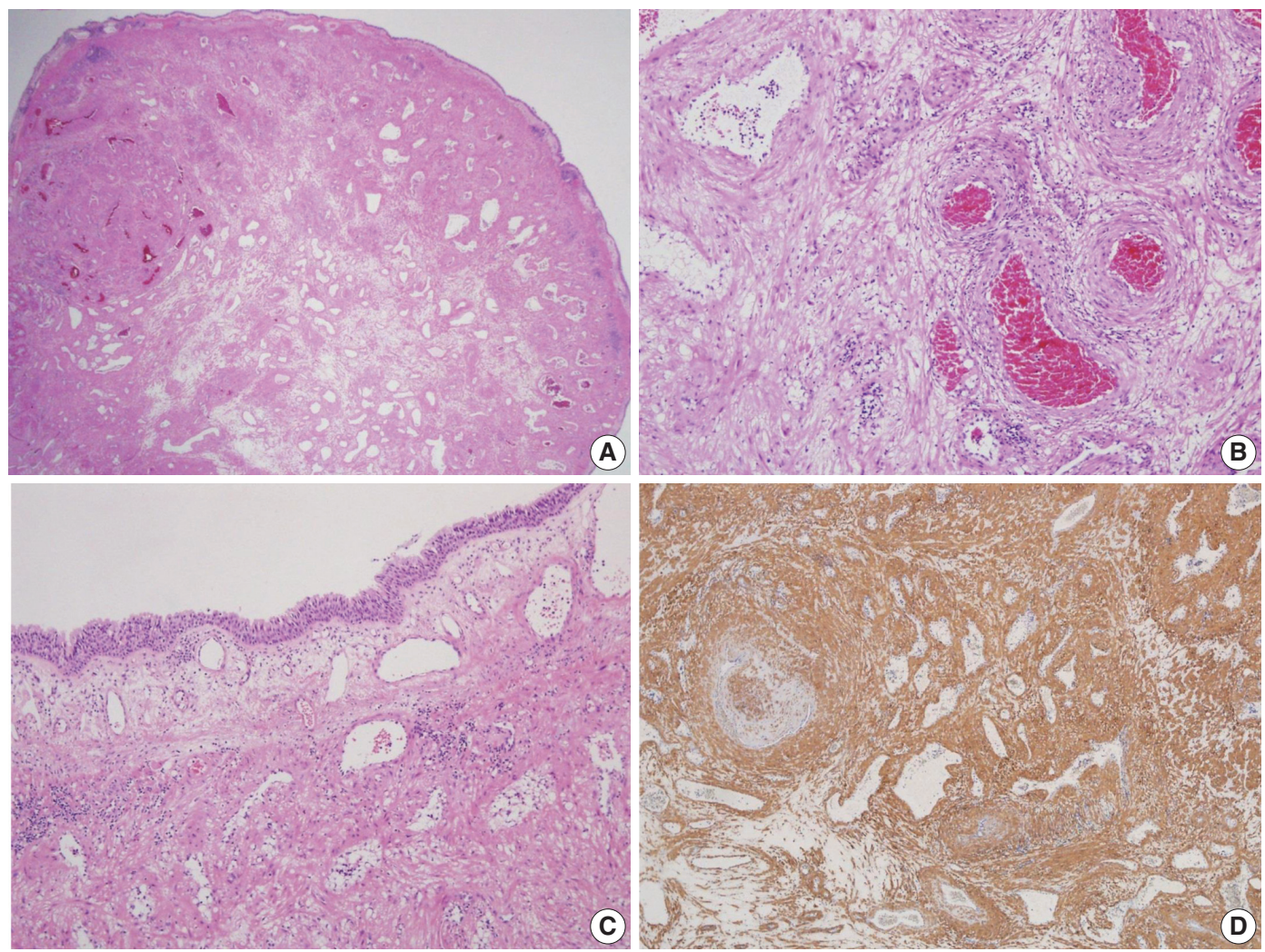

Fig. 2. Histopathologic findings of the mass. (A) Low-power view shows an ovoid and polypoid mass, consisting of well differentiated smooth muscle cells with intervening vascular channels and covered by respiratory epithelium. (B) Variable venous lumens, surrounded by muscular coats with dense to relatively loose intervascular smooth muscle cells are noted. (C) The surface is covered with ciliated pseudostratified columnar bronchial epithelium. Thin walled vessels are abundantly placed in the lamina propria, some of which have an ill-defined muscular coat. (D) Immunohistochemically, the tumor cells are positive for smooth muscle actin.

This case report was approved by the Institutional Review Boards of Catholic Medical Center Office of Human Research Protection Program (KC16ZISE0434).

\section{DISCUSSION}

Angioleiomyoma, which is a benign tumor composed of vessels and smooth muscle components, mostly affects the skin of the lower (59\%) and upper (15\%) extremities. ${ }^{2}$ It is rare in the head and neck region, accounting for $13.1 \%$ of angioleiomyomas, with mean age of 48 years and male-to-female ratio of $1.3: 1{ }^{2,3}$ Angioleiomyoma in the larynx is even less common, with about 24 total cases estimated in $2008,{ }^{4}$ with several additional cases reported in recent years. In addition, to our knowledge, there is no reported case of angioleiomyoma in the trachea. Our case revealed a lesion located near the cricoid cartilage, making it difficult to decide whether it should be characterized as being within the larynx or the trachea. According to Xu et al., ${ }^{4}$ amongst 24 cases of laryngeal angioleiomyoma, the supraglottic region was most common (14/24), followed by the glottis region (7/24) with the subglottic region (3/24) being the least common location.

Angioleiomyoma typically presents as a small, slowly growing firm nodule and generally causes pain, making this the major complaint in more than half of patients. ${ }^{1}$ In cases of laryngeal tumors, the main documented symptoms are hoarseness, dyspnea, dysphagia, and a foreign body sensation in the throat. In our case, the patient had complaints of chest discomfort, globus sensation, and throat pain. Globus sensation is presumed to be due to the mass affecting the adjacent pharynx and esophagus when swallowing or any other movement. Also throat pain is easily predictable, as most angioleiomyomas of the skin are known to frequently cause pain in the lesion. Our patient's mass was sufficiently below the vocal cord, and therefore, hoarseness was not noticed. One noteworthy point in our case is that chest discomfort with difficulty in breathing, which was one of the main complaints of the patient, was not discernible from that caused by his underlying asthma. Because of his underlying 
asthma, the patient visited the pulmonology department first, and evaluation with management was focused on asthma control for about 3 months.

A standardized treatment for laryngeal vascular leiomyoma does not exist due to its rarity, but complete mass resection is the most frequently chosen treatment. Surgical excision by both endoscope or an external approach are treatment options, with endoscopic surgery being favored due to less tissue damage. ${ }^{4}$ In this case, removal of the mass via rigid bronchoscopy, rather than by open thoracotomy, was attempted to minimize risk. The resection was performed without difficulty or any further complications. In a few previously reported cases, patients experienced expectorating fresh blood after the surgery, probably because of the abundant vascularity of the lesion. ${ }^{4,5}$ Considering its location, careful management for bleeding control and preoperative investigation of vascularity, for example using angiography, might be necessary. ${ }^{5}$

The major histological variations of angioleiomyomas are solid, venous and cavernous types, with the solid type being the most common $(67 \%))^{2}$ A study of angioleiomyoma in the head and neck also revealed that the solid variant is the most common within this location. ${ }^{3}$ Histologically, our case was venous type, showing variable venous lumina surrounded by muscular coats with relatively loose intervascular smooth muscle cells. However, histological classification as such is known to be unnecessary. Also in addition to the main mass, abundant small thin-walled vessels were noted surrounding the mass beneath the mucosa, corresponding with the gross finding of high vascularity even noticeable on the surface.

The immunohistochemical study of our case showed smooth muscle actin (+) and desmin (-) for tumor cells, which corresponds with a previous study regarding immunohistochemical findings in angioleiomyoma. ${ }^{6}$ Several studies have consistently revealed variable desmin positivity from $1 \%^{7}$ to $18 \%,{ }^{8}$ while actin is positive in every case. A more recent study revealed that the solid variant showed the highest positivity rate for desmin (75.7\%) followed by the venous type $(51.4 \%)$ and cavernous type $(18 \%)^{6}$

Angioleiomyoma is a benign disease and an extremely rare laryngeal or tracheal lesion. Despite its rarity, this disease entity should be considered when patients complain of possible symptoms, such as hoarseness, difficulty in breathing, chest discomfort or throat pain. Careful investigation might be necessary especially if the patient has underlying respiratory disease, in order to not disregard the symptoms as just being due to aggravation of an underlying disease. Angioleiomyoma is usually easily diagnosed histologically and immunohistochemically. The tumor is mostly resectable without difficulties, but careful monitoring and regular follow up is necessary due to the possibility of postoperative bleeding.

\section{Conflicts of Interest}

No potential conflict of interest relevant to this article was reported.

\section{REFERENCES}

1. Fletcher CD, Bridge JA, Hogendoorn P, Mertens F. WHO classification of tumours of soft tissue and bone. Lyon: IARC Press, 2013.

2. Hachisuga T, Hashimoto H, Enjoji M. Angioleiomyoma: a clinicopathologic reappraisal of 562 cases. Cancer 1984; 54: 126-30.

3. Wang CP, Chang YL, Sheen TS. Vascular leiomyoma of the head and neck. Laryngoscope 2004; 114: 661-5.

4. Xu Y, Zhou S, Wang S. Vascular leiomyoma of the larynx: a rare entity: three case reports and literature review. ORL J Otorhinolaryngol Relat Spec 2008; 70: 264-7.

5. Hope N, Smith CP, McCluney N. Angioleiomyoma of the larynx: beware the subglottic lesion. BMJ Case Rep 2015; 2015: bcr2015213469.

6. Matsuyama A, Hisaoka M, Hashimoto H. Angioleiomyoma: a clinicopathologic and immunohistochemical reappraisal with special reference to the correlation with myopericytoma. Hum Pathol 2007; 38: 645-51.

7. Lundgren L, Seidal T, Kindblom LG, Angervall L. Intermediate and fine filaments of vascular leiomyomas (angiomyoma), leiomyoma and leiomyosarcomas of large veins. APMIS 1989; 97: 637-45.

8. Hasegawa T, Seki K, Yang P, Hirose T, Hizawa K. Mechanism of pain and cytoskeletal properties in angioleiomyomas: an immunohistochemical study. Pathol Int 1994; 44: 66-72. 